Available online on 15.08 .2020 at http://jddtonline.info
Open Access to Pharmaceutical and Medical Research

Open $\odot$ Access

Research Article

\title{
Effectiveness of Brisk Walking Exercise on Glycaemic Control and Cardio- Vascular Risk Factors in Patients with Type 2 Diabetes
}

\author{
Dalila LAKHDAR ${ }^{1}$, Mustapha DIAF ${ }^{2 *}$, Meghit Boumediene KHALED ${ }^{3}$ \\ 1. Department of Biology and Laboratory for Research on Biological Systems and Geomatics, Faculty of Natural and Life Sciences, University \\ of Mascara, Algeria \\ 2. Department of Biology, Faculty of Natural and Life Sciences, Djillali Liabes University, Sidi-bel-Abbes, Algeria \\ 3. Department of Biology, Faculty of Natural and Life Sciences, Djillali Liabes University, Sidi-bel-Abbes, Algeria
}

\begin{abstract}
Objective: Our aim is to evaluate the effect of brisk walking exercise on anthropometric characteristics, blood parameters, physical performance and cardiovascular risk in Algerian type 2 diabetic patients.

Methods: A comparative interventional study was performed in Mascara (north-western Algeria) on patients with type 2 diabetes. All participants were subjected to a physical activity program that consisted of brisk walking sessions during seven months. Anthropometric characteristics, biochemical parameters, physical performance and cardiovascular risk indices were evaluated and compared between the two genders.

Results: Fifty-three (22 men and 31 women) type 2 diabetic patients with were involved in the study. The mean age of all participants was $50.86 \pm 11.07$ years. No difference between the two genders was observed with respect to age, anthropometrics, systolic blood pressure, maximal oxygen consumption $\left(\mathrm{VO}_{2} \mathrm{max}\right)$ and all biochemical parameters and lipid ratios. A gradual decrease in most anthropometric, clinical, biochemical parameters, and blood pressure levels have been disclosed after seven-month ( 28 weeks) of follow-up of the brisk walking programme. Higher significant decreases $(p<0.001)$ in fasting, post-effort and postprandial blood glycaemia were observed in both genders during all stages of our study. No significant differences were showed on lipid ratios; moreover, the majority of lipid ratios values were within the normal thresholds. The entirely recorded values of $\mathrm{VO}_{2} \max$, body mass index (BMI) and heart rate were slightly higher in females comparing to males with both levels of glycated haemoglobin.
\end{abstract}

Conclusion: The practice of three brisk walking sessions per week at a rate of 30 minutes each has beneficial impact on anthropometric parameters, biochemical parameters, physical performance, glycaemic control and on the reduction of cardiovascular risk in type 2 diabetic patients of both genders.

Keywords: Brisk walking, Type 2 diabetes, Glycaemic control, Blood parameters, Cardiovascular risk.

Article Info: Received 18 June 2020; Review Completed 12 July 2020; Accepted 20 July 2020; Available online 15 August 2020

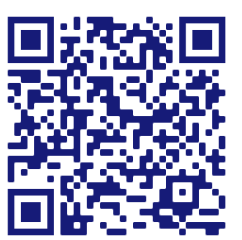

Cite this article as:

Lakhdar D, Diaf M, Khaled MB, Effectiveness of Brisk Walking Exercise on Glycaemic Control and Cardio-Vascular Risk Factors in Patients with Type 2 Diabetes, Journal of Drug Delivery and Therapeutics. 2020; 10(4-s):53-62 http://dx.doi.org/10.22270/jddt.v10i4-s.4265

Dr. DIAF Mustapha. M.Sc. PhD. Department of Biology, Faculty of Natural and Life Sciences, Djillali LIABES University, PO. BOX 89, Sidi-BelAbbes 22000, Algeria. Phone: +213 696495465 ORCID ID: https://orcid.org/0000-0001-6065-6659

\section{INTRODUCTION}

Diabetes is a group of metabolic disorders characterized by higher levels of blood glucose that result from insufficient insulin production, insulin resistance, or both. ${ }^{1}$ Since past two decades, the worldwide prevalence of type 2 diabetes (T2D) is reaching pandemic proportions. The number of individuals affected by this disease is expected to increase to $>640$ million by $2040 .^{2}$ A myriad of cardiovascular (CV) risk factors are associated with T2D and insulin resistance, and patients with T2D are generally at higher risk for atherosclerotic disorder. Therefore, CV disease is the major cause of morbidity and mortality in diabetic patients. ${ }^{3}$

The management of CV risk factors, such as hypertension and dyslipidaemia are the corn stone of the current T2D treatment strategies. ${ }^{4}$ The 2018 American Diabetes Association (ADA) guidelines emphasize the importance of an annual assessment of cardiovascular risk factors in all T2D patients. These are both traditional and non-traditional risk factors that include hypertension, dyslipidaemia, family 
history of coronary heart disease, smoking, and the presence of albuminuria. ${ }^{5}$

All strategies to improve the management of diabetic disease will involve the patient's commitment to a lifestyle change. Formal education of the diabetic patient is imperative both at the start and at regular diabetes follow-up intervals; this approach mainly concerns diet, blood sugar monitoring, issues related to pharmacotherapy, screening for complications and particularly physical activity. ${ }^{6}$

The beneficial effects of physical activity on improving human health are scientifically well established and, therefore, several international organizations such as (American College of Sports Medicine "AC SM", American Heart Association "AHA", The ADA, etc.) have developed recommendations for regular exercise for both general public and those with chronic health conditions.7,8

Current guidelines recommend moderate to vigorous aerobic exercise for patients with T2D. Exercise may include running or biking to improve physical performance. ${ }^{9}$ However, the vast majority of patients with T2D have reduced physical capacity, and are less likely to perform these physical exercises. ${ }^{10,11}$

Walking is considered a typical low impact exercise, more popular and more preferred among patients with T2D. Walking exercise is usually done at a variety of speeds with different intensities. This exercise does not require any specific skills or sophisticated assessment prior to exercise and has relatively minimal side effects. ${ }^{12}$

The results of a large a meta-analysis published in 2014 indicate that walking is associated with a decrease in glycated haemoglobin (HbA1c) and an improvement in glycaemic control in patients with T2D. A reduction in body mass index (BMI) and diastolic blood pressure (DBP) was also observed. However, the impact of walking on systolic blood pressure (SBP) and lipid profile remains inconclusive. 13 Thus, the aim of the present study was to find out the effect of 7 months (28 weeks) brisk walking exercise on anthropometric characteristics, blood parameters, physical performance and cardiovascular risk in Algerian type 2 diabetic patients.

\section{PATIENTS AND METHODS}

\section{Study population}

A total of fifty T2D patients (28 women and 22 men) were enrolled in this comparative cross-sectional study. Patients were recruited at the level of the Public Establishment for Local Health "Diabetes Centre" in the Wilaya of Mascara located in the north-western Algeria.

After obtaining the attending physician's approval and patients' consent, a close analysis of each patient's medical record was carried out in order to select solely the subjects who met our inclusion criteria that are; confirmed T2D, aged less than 74years and living in the Wilaya of Mascara. The majority of our T2D patients are treated with oral antidiabetics (mono- or dual therapy), and $1.4 \%$ were on a diet alone. The exclusion criteria consist of the presence of a confirmed cardiovascular complication, poorly controlled diabetes (blood sugar $>15 \mathrm{mmol} / \mathrm{L}$ ), untreated proliferative retinopathy, neuropathy of the lower limbs, presence of anatomical deformities or presence of lesions in the foot level.

\section{Anthropometric measurements}

Body weight (in kilograms) is determined using an

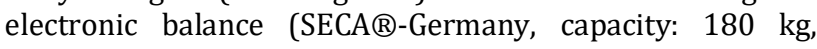

graduations $0.1 \mathrm{~kg}$ ) and height (in meters) was measured with a graduated vertical column (measuring range: 0_220 $\mathrm{cm}$, graduation length: $1 \mathrm{~mm}$ ). The BMI was calculated as weight $(\mathrm{kg}) /$ height $^{2}\left(\mathrm{~m}^{2}\right)$. Appropriate conditions have been met for weight and height measurement; patients were lightly dressed when measuring the weight and a correct position for height measuring was respected (gathered feet, straight body, heels touching the wall and looking at the horizon).

The weight measurements as well as the BMI calculation were carried out before and after the practice of the planned physical activity.

\section{Physical activity program}

The physical activity program consisted of an early morning planned brisk walking, on a $2 \mathrm{~km}$ circuit in a dedicated walkway within the Diabetes Centre, for duration of $30 \mathrm{~min}$, three times a week for seven months. We have firstly performed an electrocardiogram followed by an effort test before the first physical activity session in all patients to avoid potential issues that could arise during physical activity sessions.

The physical activity assessment was carried out each week using two different methods:

1- A subjective method using a questionnaire that includes all the activities practiced during the week; intensity, type, duration and frequency of physical activity were considered.

2- An objective method measuring heart rate using a 'Polar' type heart rate monitor. ${ }^{14,15}$

The maximum oxygen consumption $\left(\mathrm{VO}_{2} \mathrm{max}\right)$ was indirectly determined through heart rate and theoretical maximum frequency (TMF $=220$-age).

\section{Blood pressure measurement}

Blood pressure was determined using a sphygmomanometer by calculating the mean value between two consecutive measurements; the first in the lying position followed by a second one (after a few minutes) in the standing position. The blood pressure measurements were performed weekly before and after the practice of the planned physical activity.

\section{Blood sampling and assay methods}

For biochemical parameter, the blood samples were drawn in heparin tubes, between 8 and 9 AM (fasting state), after the physical activity session (post-exercise state) and $2 \mathrm{~h}$ after breakfast meal (postprandial state).

Glucose level was determined by GOD-PAP enzymatic method. The Enzymatic colorimetric methods (Test-kits Human liquicolor Germany) were used to determine total cholesterol "TC", triglycerides "TG" and high-density lipoprotein "HDL". The low-density lipoprotein "LDL" values were calculated using the Friedwald formula: $\mathrm{LDL}=\mathrm{TC}-$ HDL - TG/5 (g/L), on providing that triglycerides do not exceed $3 \mathrm{~g} / \mathrm{L}$.

Glycosylated haemoglobin (HbA1c) was measured on a whole blood sample collected on EDTA tubes. It was determined every three months during the exercise program by a chromatographic separation technique (CationExchange Resin).

\section{RESULTS}

Table 1 displays the subjects' initial characteristics. No difference between the two genders was observed with respect to age, body weight, $\mathrm{BMI}, \mathrm{SBP}, \mathrm{VO}_{2}$ max and all biochemical parameters and lipid ratios. The prevalence of 
obesity and normal weight was more pronounced in male gender; however, females were characterized by higher prevalence of overweight. A significant gender influence was noticed with regard to height, diastolic blood pressure and basic heart rate.

The evolution of the studied parameters during the seven months of the study in both genders are summarized in tables 2 and 3.We noticed a remarkable gradual decrease in most anthropometric, clinical, biochemical parameters, and the blood pressure levels after seven-month(28 weeks) of follow-up of the scheduled physical activity (brisk walking).

A higher significant decrease $(p<0.001)$ in fasting, post-effort and postprandial blood glycaemia was observed in both genders during all months of the study. The female gender recorded a significant decrease $(p=0.034)$ in the post-effort basic heart rate. On the other hand, male patients exposed a significant fluctuation ( $p=0.017$ ) in pre-effort diastolic blood pressure during the study period.
Lipid ratios (TC/HDL, LDL/HDL and TG/HDL) at the start (1 ${ }^{\text {st }}$ month) and the completion ( $7^{\text {th }}$ month) of the study were compared between males and females according to HbA1c levels (HbA1c $\leq 7 \%$ vs. $\mathrm{HbA} 1 \mathrm{c}>7 \%$ ) (figure1). The initial levels of TC/HDL and LDL/HDL ratios (during the $1^{\text {st }}$ month) were higher in all T2D male patients with both values of HbA1c (figures 1.a and 1.b). However, TG/HDL ratio was higher in women with low and high HbA1c (figure 1.c). Contrariwise, at the end of the study ( $7^{\text {th }}$ month), women with well-controlled T2D diabetes recorded higher values of TC/HDL and LDL/HDL and low TG/HDL comparing to men. Furthermore, the same observations were revealed for both men and women with higher HbA1c values.

Figure 2 shows the comparison of $\mathrm{VO}_{2} \max$ (figure 2.a), BMI (figure 2.b) and Heart Rate (figure 2.c) between males and females according to the HbA1c levels at both the beginning and the end of the physical activity program. All recorded values of $\mathrm{VO}_{2} \max , \mathrm{BMI}$ and Heart Rate were higher in females comparing to males.

Table 1: Initial characteristics of the participants

\begin{tabular}{|c|c|c|c|c|}
\hline & All patients & Males & Females & $p^{*}$ value \\
\hline$n(\%)$ & $53(100)$ & $22(41.5)$ & $31(58.5)$ & -- \\
\hline Age (years), mean \pm S.D. & $50.86 \pm 11.07$ & $52.31 \pm 9.27$ & $49.83 \pm 12.24$ & 0.427 \\
\hline \multicolumn{5}{|c|}{ Anthropometric characteristics, mean \pm S.D. } \\
\hline Weight $(\mathrm{kg})$ & $78.86 \pm 13.28$ & $81.09 \pm 10.56$ & $77.29 \pm 14.89$ & 0.310 \\
\hline Height (m) & $1.65 \pm 0.08$ & $1.72 \pm 0.06$ & $1.61 \pm 0.05$ & $<0.001$ \\
\hline BMI $\left(\mathrm{kg} / \mathrm{m}^{2}\right)$ & $28.80 \pm 4.97$ & $27.83 \pm 3.92$ & $29.50 \pm 5.56$ & 0.232 \\
\hline \multicolumn{5}{|c|}{ Prevalence of weight categories, $\boldsymbol{n}(\%)$} \\
\hline Normal weight, $\mathrm{BMI}<25 \mathrm{~kg} / \mathrm{m}^{2}$ & $12(22.6)$ & $5(9.4)$ & $7(13.2)$ & \multirow{3}{*}{0.229} \\
\hline Overweight, $\mathrm{BMI}=25.0-29.9 \mathrm{~kg} / \mathrm{m}^{2}$ & $20(37.7)$ & $11(20.7)$ & $9(17.0)$ & \\
\hline Obesity, BMI $\geq 30 \mathrm{~kg} / \mathrm{m}^{2}$ & $21(39.6)$ & $6(11.3)$ & $15(28.3)$ & \\
\hline \multicolumn{5}{|l|}{ Blood pressure, mean \pm S.D. } \\
\hline Systolic pressure (mmHg) & $134.71 \pm 24.54$ & $130.90 \pm 22.44$ & $137.41 \pm 25.94$ & 0.346 \\
\hline Diastolic pressure (mmHg) & $63.39 \pm 20.18$ & $55.90 \pm 21.30$ & $68.70 \pm 17.84$ & 0.021 \\
\hline \multicolumn{5}{|l|}{ Clinical parameters, mean \pm S.D. } \\
\hline Basic heart rate (beats/minutes) & $82.71 \pm 11.75$ & $77.45 \pm 11.90$ & $86.45 \pm 10.27$ & 0.005 \\
\hline $\mathrm{VO}_{2} \max (\%)$ & $54.86 \pm 7.63$ & $52.72 \pm 7.49$ & $56.38 \pm 7.49$ & 0.086 \\
\hline Theoretical maximum heart rate & $168.60 \pm 12.09$ & $166.73 \pm 11.55$ & $169.93 \pm 12.32$ & 0.012 \\
\hline \multicolumn{5}{|l|}{ Biochemical parameters, mean \pm S.D. } \\
\hline Fasting glycaemia $(\mathrm{g} / \mathrm{L})$ & $2.19 \pm 0.60$ & $2.13 \pm 0.66$ & $2.24 \pm 0.55$ & 0.493 \\
\hline Post-prandial glycaemia (g/L) & $2.34 \pm 0.81$ & $2.34 \pm 0.76$ & $2.34 \pm 0.85$ & 0.997 \\
\hline $\operatorname{HbA1c}(\%)$ & $10.17 \pm 2.79$ & $9.99 \pm 2.88$ & $10.29 \pm 2.76$ & 0.696 \\
\hline Total cholesterol (g/L) & $1.82 \pm 0.44$ & $1.80 \pm 0.44$ & $1.82 \pm 0.44$ & 0.876 \\
\hline HDL (g/L) & $0.49 \pm 0.06$ & $0.47 \pm 0.06$ & $0.50 \pm 0.06$ & 0.146 \\
\hline LDL $(\mathrm{g} / \mathrm{L})$ & $1.02 \pm 0.46$ & $1.00 \pm 0.47$ & $1.03 \pm 0.46$ & 0.787 \\
\hline Triglycerides (g/L) & $1.53 \pm 0.73$ & $1.66 \pm 0.98$ & $1.44 \pm 0.48$ & 0.294 \\
\hline Urea $(\mathrm{g} / \mathrm{L})$ & $0.26 \pm 0.08$ & $0.28 \pm 0.08$ & $0.25 \pm 0.07$ & 0.223 \\
\hline Creatinine $(\mathrm{g} / \mathrm{L})$ & $9.88 \pm 2.07$ & $10.11 \pm 1.79$ & $9.37 \pm 2.25$ & 0.529 \\
\hline \multicolumn{5}{|l|}{ Lipid ratios } \\
\hline $\mathrm{TC} / \mathrm{HDL}$ & $3.79 \pm 1.09$ & $3.88 \pm 1.07$ & $3.72 \pm 1.12$ & 0.605 \\
\hline LDL/HDL & $2.14 \pm 1.05$ & $2.16 \pm 1.06$ & $2.13 \pm 1.06$ & 0.930 \\
\hline TG/HDL & $3.22 \pm 1.68$ & $3.61 \pm 2.24$ & $2.94 \pm 1.09$ & 0.152 \\
\hline
\end{tabular}

(*) Comparison between males and females; mean values were compared using Student's $t$-test. Percentages were compared with Chi-square test. S.D.: standard deviation. BMI: body mass index. $\mathbf{V O}_{2}$ max: maximal oxygen consumption. HbA1c: glycated hemoglobin. HDL: high-density lipoprotein. LDL: low-density lipoprotein. TC: total cholesterol. TG: triglycerides. 
Table 2: Evolution of the studied parameters during the seven months of the study in women

\begin{tabular}{|c|c|c|c|c|c|c|c|c|c|}
\hline & $\begin{array}{l}1^{\text {st }} \text { month } \\
\text { (mean } \pm S \text {. } \\
\text { D.) }\end{array}$ & $\begin{array}{l}2^{\text {nd }} \text { month } \\
\text { (mean } \pm \text { S. } \\
\text { D.) }\end{array}$ & $\begin{array}{l}3^{\text {rd }} \text { month } \\
(\text { mean } \pm \text { S.D } \\
.)\end{array}$ & $\begin{array}{l}4^{\text {th }} \text { month } \\
\text { (mean } \pm \text { S.D } \\
.)\end{array}$ & $\begin{array}{l}5^{\text {th }} \text { month } \\
(\text { mean } \pm \text { S.D } \\
.)\end{array}$ & $\begin{array}{l}6^{\text {th }} \text { month } \\
\text { (mean } \pm \text { S.D } \\
.)\end{array}$ & $\begin{array}{l}7^{\text {th }} \text { month } \\
(\text { mean } \pm \text { S.D } \\
.)\end{array}$ & $\begin{array}{c}\chi^{2} \\
\text { Chi-2 } \\
\text { test }\end{array}$ & $p^{*}$ \\
\hline Weight (kg) & $\begin{array}{c}77.29 \pm 14 \\
89\end{array}$ & $\begin{array}{c}76.40 \pm 14 \\
94\end{array}$ & $\begin{array}{c}75.54 \pm 15 \\
16\end{array}$ & $\begin{array}{c}75.00 \pm 14 \\
92\end{array}$ & $\begin{array}{c}74.38 \pm 14 \\
65\end{array}$ & $\begin{array}{c}74.06 \pm 14 \\
55\end{array}$ & $\begin{array}{c}73.19 \pm 14 \\
52\end{array}$ & 2.760 & 0.838 \\
\hline BMI $\left(\mathrm{kg} / \mathrm{m}^{2}\right)$ & $\begin{array}{c}29.50 \pm 5.5 \\
6\end{array}$ & $\begin{array}{c}29.16 \pm 5.6 \\
7\end{array}$ & $\begin{array}{c}28.82 \pm 5.6 \\
9\end{array}$ & $\begin{array}{c}28.65 \pm 5.6 \\
1\end{array}$ & $\begin{array}{c}28.40 \pm 5.4 \\
6\end{array}$ & $\begin{array}{c}28.27 \pm 5.4 \\
3\end{array}$ & $\begin{array}{c}27.95 \pm 5.4 \\
5\end{array}$ & 2.475 & 0.871 \\
\hline $\begin{array}{l}\text { Systolic pressure } \\
\text { pre-effort } \\
(\mathrm{mmHg})\end{array}$ & $\begin{array}{c}137.41 \pm 25 \\
.94\end{array}$ & $\begin{array}{c}126.19 \pm 27 \\
.85\end{array}$ & $\begin{array}{c}129.35 \pm 21 \\
.43\end{array}$ & $\begin{array}{c}134.19 \pm 22 \\
.17\end{array}$ & $\begin{array}{c}137.41 \pm 16 \\
.72\end{array}$ & $\begin{array}{c}136.12 \pm 17 \\
.82\end{array}$ & $\begin{array}{c}137.41 \pm 19 \\
.48\end{array}$ & 6.400 & 0.380 \\
\hline $\begin{array}{l}\text { Diastolic } \\
\text { pressure pre- } \\
\text { effort (mmHg) }\end{array}$ & $\begin{array}{c}68.70 \pm 17 \\
84\end{array}$ & $\begin{array}{c}60.32 \pm 14 \\
48\end{array}$ & $\begin{array}{c}65.80 \pm 11 \\
48\end{array}$ & $\begin{array}{c}63.54 \pm 9.8 \\
4\end{array}$ & $\begin{array}{c}65.48 \pm 16 \\
89\end{array}$ & $\begin{array}{c}64.19 \pm 8.0 \\
7\end{array}$ & $\begin{array}{c}62.90 \pm 9.7 \\
2\end{array}$ & 9.699 & 0.138 \\
\hline $\begin{array}{l}\text { Systolic pressure } \\
\text { post-effort } \\
(\mathrm{mmHg})\end{array}$ & $\begin{array}{c}122.32 \pm 18 \\
.66\end{array}$ & $\begin{array}{c}119.96 \pm 20 \\
.54\end{array}$ & $\begin{array}{c}116.58 \pm 19 \\
.17\end{array}$ & $\begin{array}{c}121.93 \pm 21 \\
.04\end{array}$ & $\begin{array}{c}124.51 \pm 16 \\
.29\end{array}$ & $\begin{array}{c}121.93 \pm 16 \\
.00\end{array}$ & $\begin{array}{c}121.61 \pm 16 \\
.95\end{array}$ & 3.475 & 0.747 \\
\hline $\begin{array}{l}\text { Diastolic } \\
\text { pressure post- } \\
\text { effort ( } \mathrm{mmHg} \text { ) }\end{array}$ & $\begin{array}{c}62.80 \pm 18 \\
35\end{array}$ & $\begin{array}{c}53.61 \pm 19 . \\
02\end{array}$ & $\begin{array}{l}58.70 \pm 12 . \\
\quad 64\end{array}$ & $\begin{array}{c}58.77 \pm 12 \\
18\end{array}$ & $\begin{array}{c}59.80 \pm 12 \\
08\end{array}$ & $\begin{array}{c}56.87 \pm 9.2 \\
0\end{array}$ & $\begin{array}{c}57.80 \pm 10 . \\
72\end{array}$ & 5.895 & 0.435 \\
\hline $\begin{array}{l}\text { Basic heart rate } \\
\text { pre- } \\
\text { effort(beats/min } \\
\text { utes) }\end{array}$ & $\begin{array}{c}86.45 \pm 10 . \\
27\end{array}$ & $\begin{array}{c}83.19 \pm 11 . \\
98\end{array}$ & $\begin{array}{c}81.41 \pm 10 . \\
83\end{array}$ & $\begin{array}{c}84.70 \pm 9.1 \\
1\end{array}$ & $\begin{array}{c}83.83 \pm 11 \\
52\end{array}$ & $\begin{array}{c}84.67 \pm 8.0 \\
2\end{array}$ & $\begin{array}{c}83.80 \pm 8.9 \\
4\end{array}$ & 6.026 & 0.420 \\
\hline $\begin{array}{l}\text { Basic heart rate } \\
\text { post- } \\
\text { effort(beats/min } \\
\text { utes) }\end{array}$ & $\begin{array}{l}95.32 \pm 10 \\
97\end{array}$ & $\begin{array}{c}90.70 \pm 12 \\
36\end{array}$ & $\begin{array}{c}88.61 \pm 9.6 \\
7\end{array}$ & $\begin{array}{c}92.19 \pm 9.9 \\
3\end{array}$ & $\begin{array}{c}92.70 \pm 10 \\
83\end{array}$ & $\begin{array}{c}95.00 \pm 8.0 \\
4\end{array}$ & $\begin{array}{c}92.25 \pm 6.9 \\
0\end{array}$ & $\begin{array}{c}13.64 \\
7\end{array}$ & 0.034 \\
\hline $\mathrm{VO}_{2} \max (\%)$ & $\begin{array}{c}56.38 \pm 7.4 \\
9\end{array}$ & $\begin{array}{c}53.56 \pm 7.3 \\
7\end{array}$ & $\begin{array}{c}52.42 \pm 6.7 \\
0\end{array}$ & $\begin{array}{c}54.52 \pm 6.9 \\
3\end{array}$ & $\begin{array}{c}54.81 \pm 7.0 \\
4\end{array}$ & $\begin{array}{c}56.13 \pm 5.5 \\
4\end{array}$ & $\begin{array}{c}54.54 \pm 5.1 \\
7\end{array}$ & 6.412 & 0.379 \\
\hline $\begin{array}{l}\text { Fasting } \\
\text { glycaemia (g/L) }\end{array}$ & $2.24 \pm 0.55$ & $2.02 \pm 0.61$ & $1.88 \pm 0.52$ & $1.97 \pm 0.59$ & $1.91 \pm 0.54$ & $1.88 \pm 0.42$ & $1.62 \pm 0.23$ & $\begin{array}{c}23.95 \\
8\end{array}$ & 0.010 \\
\hline $\begin{array}{l}\text { Glycaemia post- } \\
\text { effort }(\mathrm{g} / \mathrm{L})\end{array}$ & $2.25 \pm 0.91$ & $2.04 \pm 0.63$ & $1.75 \pm 0.53$ & $1.73 \pm 0.72$ & $1.60 \pm 0.36$ & $1.54 \pm 0.22$ & $1.45 \pm 0.20$ & $\begin{array}{c}35.77 \\
9\end{array}$ & $\begin{array}{c}<0.00 \\
1\end{array}$ \\
\hline $\begin{array}{l}\text { Post-prandial } \\
\text { glycaemia }(\mathrm{g} / \mathrm{L})\end{array}$ & $2.34 \pm 0.85$ & $1.96 \pm 0.53$ & $1.71 \pm 0.33$ & $1.76 \pm 0.66$ & $1.60 \pm 0.38$ & $1.55 \pm 0.26$ & $1.45 \pm 0.20$ & $\begin{array}{c}52.39 \\
7\end{array}$ & $\begin{array}{c}<0.00 \\
1\end{array}$ \\
\hline HbA1c (\%) & $\begin{array}{c}10.29 \pm 2.7 \\
6\end{array}$ & -- & -- & $9.87 \pm 2.72$ & -- & -- & $9.32 \pm 2.89$ & 5.117 & 0.077 \\
\hline $\begin{array}{l}\text { Total cholesterol } \\
\text { (g/L) }\end{array}$ & $1.82 \pm 0.44$ & $1.84 \pm 0.40$ & $1.74 \pm 0.46$ & $1.78 \pm 0.42$ & $1.75 \pm 0.65$ & $1.67 \pm 0.31$ & $1.66 \pm 0.24$ & 8.242 & 0.221 \\
\hline HDL (g/L) & $0.50 \pm 0.06$ & $0.49 \pm 0.08$ & $0.49 \pm 0.06$ & $0.50 \pm 0.07$ & $0.50 \pm 0.09$ & $0.50 \pm 0.08$ & $0.50 \pm 0.07$ & 1.673 & 0.947 \\
\hline LDL (g/L) & $1.03 \pm 0.46$ & $1.05 \pm 0.44$ & $0.96 \pm 0.46$ & $1.02 \pm 0.44$ & $0.98 \pm 0.65$ & $0.90 \pm 0.32$ & $0.90 \pm 0.24$ & 5.687 & 0.459 \\
\hline $\begin{array}{l}\text { Triglycerides } \\
\text { (g/L) }\end{array}$ & $1.44 \pm 0.48$ & $1.44 \pm 0.47$ & $1.43 \pm 0.56$ & $1.29 \pm 0.26$ & $1.31 \pm 0.26$ & $1.30 \pm 0.18$ & $1.28 \pm 0.22$ & 6.922 & 0.328 \\
\hline Urea $(\mathrm{g} / \mathrm{L})$ & $0.25 \pm 0.07$ & $0.23 \pm 0.06$ & $0.24 \pm 0.07$ & $0.22 \pm 0.06$ & $0.24 \pm 0.07$ & $0.26 \pm 0.07$ & $0.25 \pm 0.06$ & 4.477 & 0.612 \\
\hline Creatinine (g/L) & $9.73 \pm 2.25$ & $9.33 \pm 1.18$ & $9.35 \pm 1.17$ & $9.39 \pm 1.09$ & $9.01 \pm 0.92$ & $9.27 \pm 1.07$ & $9.10 \pm 0.92$ & 2.573 & 0.860 \\
\hline TC/HDL & $3.72 \pm 1.12$ & $3.87 \pm 1.29$ & $3.62 \pm 1.10$ & $3.66 \pm 1.23$ & $3.70 \pm 2.16$ & $3.38 \pm 0.88$ & $3.37 \pm 0.75$ & 4.306 & 0.635 \\
\hline LDL/HDL & $2.13 \pm 1.06$ & $2.26 \pm 1.17$ & $2.02 \pm 1.04$ & $2.13 \pm 1.12$ & $2.15 \pm 1.97$ & $1.85 \pm 0.82$ & $1.85 \pm 0.67$ & 3.820 & 0.701 \\
\hline TG/HDL & $2.94 \pm 1.09$ & $3.02 \pm 1.28$ & $3.00 \pm 1.37$ & $2.66 \pm 0.90$ & $2.74 \pm 1.01$ & $2.63 \pm 0.59$ & $2.57 \pm 0.56$ & 4.246 & 0.643 \\
\hline
\end{tabular}

(*) Comparison of the parameters during the seven months of the study using ANOVA Kruskal-Wallis test. S.D.: standard deviation. BMI: body mass index. $\mathbf{V O}_{2}$ max: maximal oxygen consumption. HbA1c: glycated hemoglobin. HDL: high-density lipoprotein. LDL: low-density lipoprotein. TC: total cholesterol. TG: triglycerides. 
Table 3: Evolution of the studied parameters during the seven months of the study in men

\begin{tabular}{|c|c|c|c|c|c|c|c|c|c|}
\hline & $\begin{array}{l}1^{\text {st }} \text { month } \\
\text { (mean } \pm \text { S. } \\
\text { D.) }\end{array}$ & $\begin{array}{l}2^{\text {nd }} \text { month } \\
\text { (mean } \pm \text { S. } \\
\text { D.) }\end{array}$ & $\begin{array}{l}\text { 3rd month } \\
\text { (mean } \pm \text { S. } \\
\text { D.) }\end{array}$ & $\begin{array}{l}4^{\text {th }} \text { month } \\
\text { (mean } \pm \text { S. } \\
\text { D.) }\end{array}$ & $\begin{array}{l}5^{\text {th }} \text { month } \\
\text { (mean } \pm S \text {. } \\
\text { D.) }\end{array}$ & $\begin{array}{l}6^{\text {th }} \text { month } \\
\text { (mean } \pm S \text {. } \\
\text { D.) }\end{array}$ & $\begin{array}{l}7^{\text {th }} \text { month } \\
\text { (mean } \pm \text { S. } \\
\text { D.) }\end{array}$ & $\begin{array}{c}\chi^{2} \\
\text { Chi-2 } \\
\text { test }\end{array}$ & $p^{*}$ \\
\hline Weight (kg) & $\begin{array}{c}81.09 \pm 10 . \\
56\end{array}$ & $\begin{array}{c}79.81 \pm 10 . \\
43\end{array}$ & $\begin{array}{c}79.18 \pm 10 . \\
18\end{array}$ & $\begin{array}{c}78.68 \pm 10 . \\
74\end{array}$ & $\begin{array}{c}77.95 \pm 10 . \\
22\end{array}$ & $\begin{array}{c}77.50 \pm 10 . \\
36\end{array}$ & $\begin{array}{c}76.95 \pm 10 . \\
45\end{array}$ & 3.172 & 0.787 \\
\hline BMI $\left(\mathrm{kg} / \mathrm{m}^{2}\right)$ & $\begin{array}{c}27.83 \pm 3.9 \\
2\end{array}$ & $\begin{array}{c}27.44 \pm 3.7 \\
0\end{array}$ & $\begin{array}{c}27.23 \pm 3.6 \\
2\end{array}$ & $\begin{array}{c}27.13 \pm 3.7 \\
9\end{array}$ & $\begin{array}{c}26.85 \pm 3.5 \\
6\end{array}$ & $\begin{array}{c}26.69 \pm 3.6 \\
2\end{array}$ & $\begin{array}{c}26.51 \pm 3.6 \\
1\end{array}$ & 2.957 & 0.814 \\
\hline $\begin{array}{l}\text { Systolic } \\
\text { pressure pre- } \\
\text { effort (mmHg) }\end{array}$ & $\begin{array}{c}130.90 \pm 2 \\
2.44\end{array}$ & $\begin{array}{c}130.45 \pm 2 \\
5.53\end{array}$ & $\begin{array}{c}128.63 \pm 2 \\
2.94\end{array}$ & $\begin{array}{c}131.36 \pm 1 \\
5.82\end{array}$ & $\begin{array}{c}131.36 \pm 1 \\
2.06\end{array}$ & $\begin{array}{c}135.00 \pm 1 \\
6.54\end{array}$ & $\begin{array}{c}133.18 \pm 1 \\
0.41\end{array}$ & 3.056 & 0.802 \\
\hline $\begin{array}{l}\text { Diastolic } \\
\text { pressure pre- } \\
\text { effort (mmHg) }\end{array}$ & $\begin{array}{c}55.90 \pm 21 . \\
30\end{array}$ & $\begin{array}{c}67.72 \pm 14 \\
45\end{array}$ & $\begin{array}{c}64.09 \pm 8.5 \\
4\end{array}$ & $\begin{array}{c}67.72 \pm 11 \\
09\end{array}$ & $\begin{array}{c}68.63 \pm 9.4 \\
0\end{array}$ & $\begin{array}{c}68.63 \pm 10 \\
37\end{array}$ & $\begin{array}{c}63.18 \pm 7.7 \\
9\end{array}$ & 15.521 & 0.017 \\
\hline $\begin{array}{l}\text { Systolic } \\
\text { pressure post- } \\
\text { effort (mmHg) }\end{array}$ & $\begin{array}{c}122.72 \pm 2 \\
3.13\end{array}$ & $\begin{array}{c}118.27 \pm 3 \\
1.09\end{array}$ & $\begin{array}{c}122.72 \pm 2 \\
6.93\end{array}$ & $\begin{array}{c}121.36 \pm 1 \\
7.80\end{array}$ & $\begin{array}{c}124.54 \pm 1 \\
7.38\end{array}$ & $\begin{array}{c}119.54 \pm 1 \\
5.57\end{array}$ & $\begin{array}{c}120.90 \pm 1 \\
2.69\end{array}$ & 0.704 & 0.994 \\
\hline $\begin{array}{l}\text { Diastolic } \\
\text { pressure post- } \\
\text { effort (mmHg) }\end{array}$ & $\begin{array}{c}64.09 \pm 21 \\
74\end{array}$ & $\begin{array}{c}60.45 \pm 21 \\
\quad 92\end{array}$ & $\begin{array}{c}60.90 \pm 15 \\
40\end{array}$ & $\begin{array}{c}60.45 \pm 12 \\
52\end{array}$ & $\begin{array}{c}58.18 \pm 15 \\
62\end{array}$ & $\begin{array}{c}58.18 \pm 12 . \\
58\end{array}$ & $\begin{array}{c}56.81 \pm 10 . \\
41\end{array}$ & 2.343 & 0.886 \\
\hline $\begin{array}{l}\text { Basic heart rate } \\
\text { pre- } \\
\text { effort(beats/mi } \\
\text { nutes) }\end{array}$ & $\begin{array}{c}77.45 \pm 11 . \\
90\end{array}$ & $\begin{array}{c}78.27 \pm 12 . \\
91\end{array}$ & $\begin{array}{c}75.31 \pm 11 . \\
29\end{array}$ & $\begin{array}{c}74.86 \pm 8.6 \\
0\end{array}$ & $\begin{array}{c}75.50 \pm 10 \\
30\end{array}$ & $\begin{array}{c}76.27 \pm 8.5 \\
8\end{array}$ & $\begin{array}{c}75.59 \pm 9.4 \\
2\end{array}$ & 1.651 & 0.949 \\
\hline $\begin{array}{l}\text { Basic heart rate } \\
\text { post- } \\
\text { effort(beats/mi } \\
\text { nutes) }\end{array}$ & $\begin{array}{c}86.31 \pm 12 . \\
55\end{array}$ & $\begin{array}{c}88.36 \pm 12 . \\
37\end{array}$ & $\begin{array}{c}82.63 \pm 12 . \\
80\end{array}$ & $\begin{array}{c}84.27 \pm 10 \\
30\end{array}$ & $\begin{array}{c}82.90 \pm 10 . \\
56\end{array}$ & $\begin{array}{c}85.40 \pm 10 . \\
90\end{array}$ & $\begin{array}{c}85.04 \pm 10 . \\
69\end{array}$ & 4.505 & 0.609 \\
\hline $\mathrm{VO}_{2} \max (\%)$ & $\begin{array}{c}52.72 \pm 7.4 \\
9\end{array}$ & $\begin{array}{c}55.36 \pm 12 . \\
38\end{array}$ & $\begin{array}{c}49.98 \pm 7.7 \\
7\end{array}$ & $\begin{array}{c}51.37 \pm 5.9 \\
5\end{array}$ & $\begin{array}{c}50.31 \pm 5.8 \\
6\end{array}$ & $\begin{array}{c}51.98 \pm 6.8 \\
5\end{array}$ & $\begin{array}{c}51.48 \pm 6.3 \\
4\end{array}$ & 4.066 & 0.668 \\
\hline $\begin{array}{l}\text { Fasting } \\
\text { glycaemia }(\mathrm{g} / \mathrm{L})\end{array}$ & $2.13 \pm 0.66$ & $1.95 \pm 0.66$ & $1.81 \pm 0.62$ & $1.88 \pm 0.44$ & $1.90 \pm 0.51$ & $1.69 \pm 0.35$ & $1.50 \pm 0.23$ & 16.611 & 0.011 \\
\hline $\begin{array}{l}\text { Glycaemia post- } \\
\text { effort }(\mathrm{g} / \mathrm{L})\end{array}$ & $2.13 \pm 0.70$ & $2.17 \pm 0.84$ & $1.82 \pm 0.78$ & $1.55 \pm 0.37$ & $1.60 \pm 0.39$ & $1.47 \pm 0.23$ & $1.38 \pm 0.20$ & 25.966 & $\begin{array}{c}<0.00 \\
1\end{array}$ \\
\hline $\begin{array}{l}\text { Post-prandial } \\
\text { glycaemia }(\mathrm{g} / \mathrm{L})\end{array}$ & $2.34 \pm 0.76$ & $1.93 \pm 0.73$ & $1.70 \pm 0.63$ & $1.57 \pm 0.23$ & $1.55 \pm 0.33$ & $1.55 \pm 0.20$ & $1.47 \pm 0.18$ & 24.929 & $\begin{array}{c}<0.00 \\
1\end{array}$ \\
\hline HbA1c (\%) & $9.99 \pm 2.88$ & -- & -- & $9.58 \pm 3.08$ & -- & -- & $8.99 \pm 2.45$ & 2.605 & 0.272 \\
\hline $\begin{array}{l}\text { Total cholesterol } \\
\text { (g/L) }\end{array}$ & $1.80 \pm 0.44$ & $1.57 \pm 0.27$ & $1.73 \pm 0.44$ & $1.63 \pm 0.45$ & $1.72 \pm 0.45$ & $1.74 \pm 0.40$ & $1.59 \pm 0.29$ & 4.677 & 0.586 \\
\hline HDL (g/L) & $0.47 \pm 0.06$ & $0.49 \pm 0.06$ & $0.48 \pm 0.05$ & $0.49 \pm 0.05$ & $0.47 \pm 0.06$ & $0.47 \pm 0.06$ & $0.48 \pm 0.06$ & 3.360 & 0.762 \\
\hline LDL (g/L) & $1.00 \pm 0.47$ & $0.79 \pm 0.33$ & $0.96 \pm 0.46$ & $0.89 \pm 0.44$ & $1.02 \pm 0.47$ & $1.00 \pm 0.38$ & $0.86 \pm 0.31$ & 4.317 & 0.634 \\
\hline $\begin{array}{l}\text { Triglycerides } \\
\text { (g/L) }\end{array}$ & $1.66 \pm 0.98$ & $1.39 \pm 0.51$ & $1.38 \pm 0.53$ & $1.24 \pm 0.28$ & $1.14 \pm 0.26$ & $1.29 \pm 0.40$ & $1.25 \pm 0.22$ & 2.903 & 0.821 \\
\hline Urea $(\mathrm{g} / \mathrm{L})$ & $0.28 \pm 0.08$ & $0.28 \pm 0.07$ & $0.30 \pm 0.16$ & $0.29 \pm 0.07$ & $0.29 \pm 0.07$ & $0.27 \pm 0.05$ & $0.28 \pm 0.05$ & 1.701 & 0.945 \\
\hline Creatinine $(\mathrm{g} / \mathrm{L})$ & $\begin{array}{c}10.11 \pm 1.7 \\
9\end{array}$ & $9.31 \pm 1.07$ & $9.33 \pm 1.87$ & $9.12 \pm 1.14$ & $9.24 \pm 0.94$ & $9.23 \pm 0.97$ & $8.71 \pm 0.64$ & 10.720 & 0.097 \\
\hline $\mathrm{TC} / \mathrm{HDL}$ & $3.88 \pm 1.07$ & $3.24 \pm 0.77$ & $3.65 \pm 1.23$ & $3.33 \pm 0.95$ & $3.73 \pm 1.24$ & $3.75 \pm 1.17$ & $3.39 \pm 0.92$ & 6.568 & 0.363 \\
\hline LDL/HDL & $2.16 \pm 1.06$ & $1.66 \pm 0.78$ & $2.07 \pm 1.21$ & $1.82 \pm 0.92$ & $2.24 \pm 1.22$ & $2.19 \pm 1.04$ & $1.85 \pm 0.85$ & 5.096 & 0.532 \\
\hline TG/HDL & $3.61 \pm 2.24$ & $2.89 \pm 1.21$ & $2.88 \pm 1.07$ & $2.55 \pm 0.67$ & $2.47 \pm 0.70$ & $2.78 \pm 1.02$ & $2.67 \pm 0.71$ & 3.054 & 0.802 \\
\hline
\end{tabular}

(*) Comparison of the parameters during the seven months of the study using ANOVA Kruskal-Wallis test. S.D.: standard deviation. BMI: body mass index. $\mathbf{V O}_{2}$ max: maximal oxygen consumption. HbA1c: glycated hemoglobin. HDL: high-density lipoprotein. LDL: low-density lipoprotein. TC: total cholesterol. TG: triglycerides. 


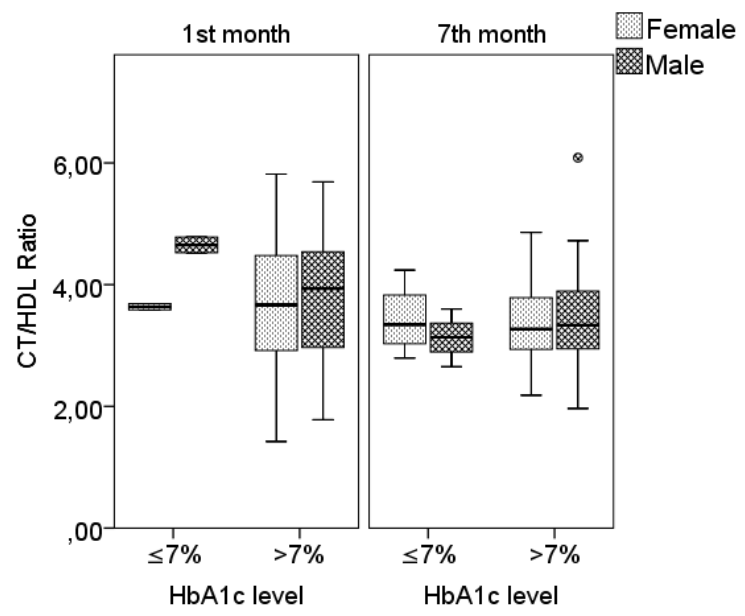

(1.a)

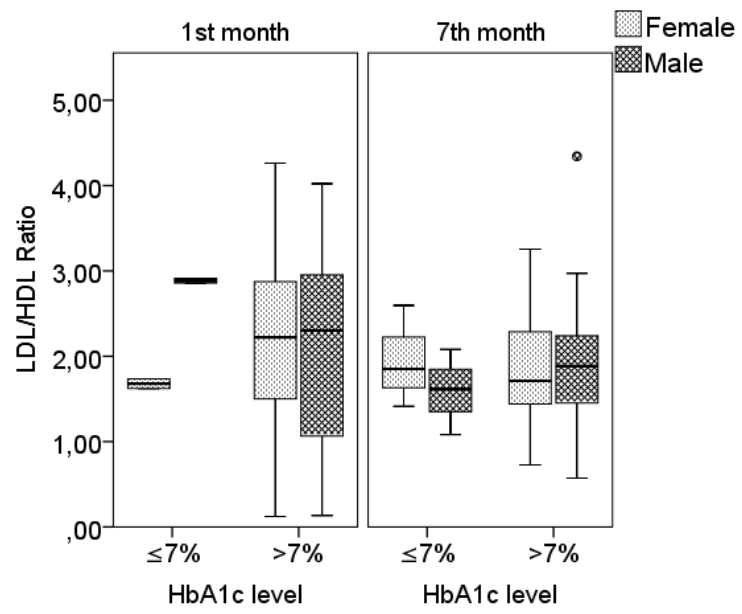

(1.b)

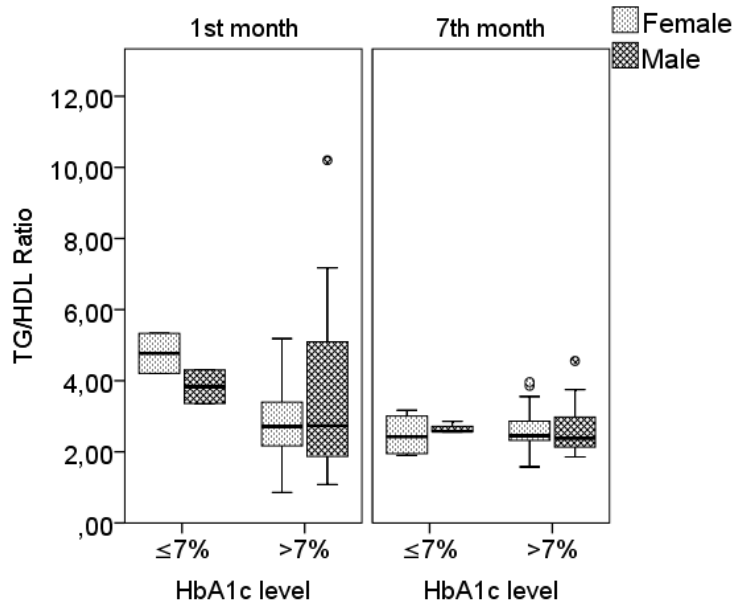

(1.c)

Figure 1: Comparison of the $1^{\text {st }}$ and the $7^{\text {th }}$ months' lipid ratios levels between the two genders according to HbA1c levels 


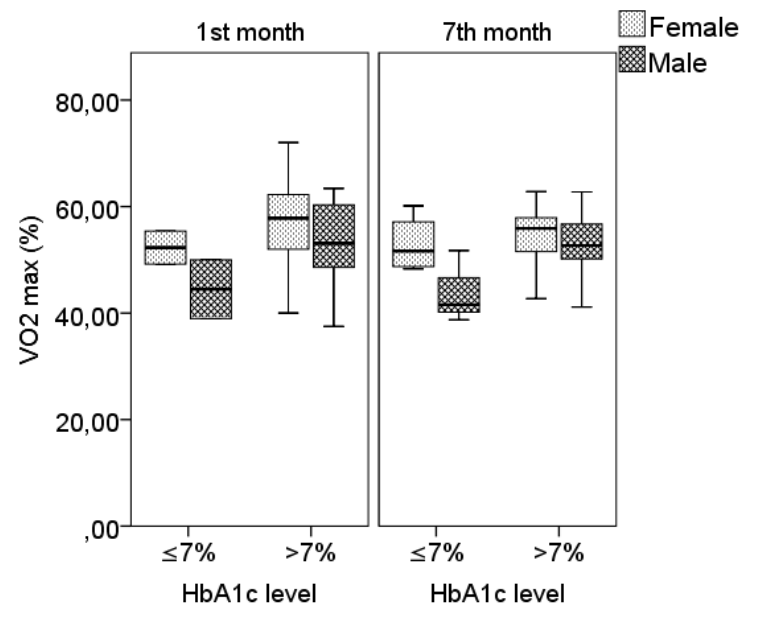

(2.a)

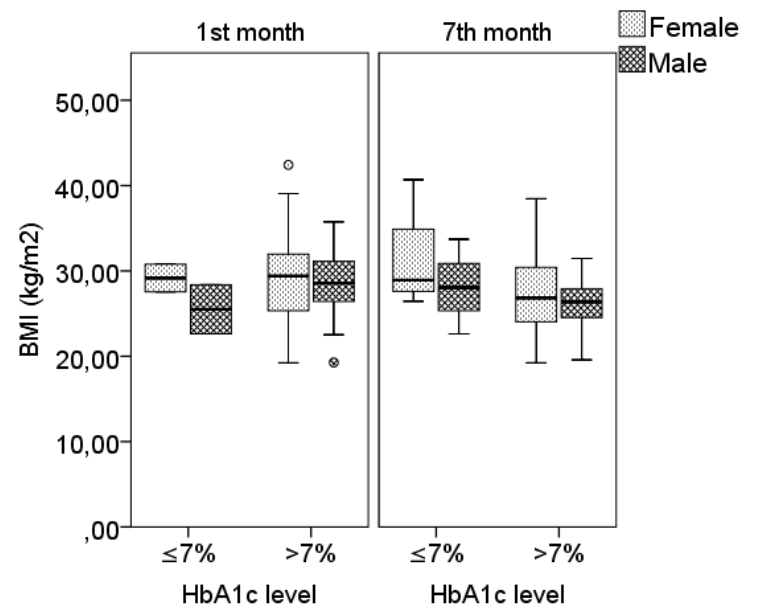

(2.b)

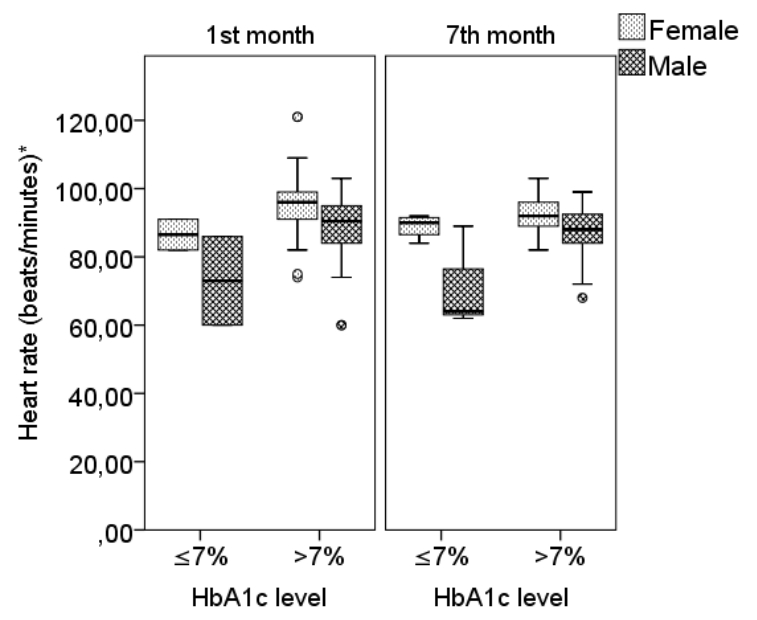

(2.c)

Figure 2: Comparison of the $1^{\text {st }}$ and the $7^{\text {th }}$ months' $\mathrm{VO}_{2}$ maw, $\mathrm{BMI}$ and Heart rate between the two genders according to $\mathrm{HbA1c}$ levels 


\section{DISCUSSION}

The three major elements of good T2D therapy have long been based on regular exercise along with diet and antidiabetic medications. Structured physical activity programs have been shown to be as effective as pharmaceutical strategies for improving both glycaemic control and cardiovascular risk profile. ${ }^{16,17}$ Most intervention studies on physical activities in diabetics use endurance and resistance type exercises supervised by a physical therapist. ${ }^{18,19}$ The brisk walking exercise represents a less expensive alternative that often gives good clinical results when the patients are well supervised. ${ }^{20}$

In the present study, we compared the health benefits of seven months brisk walking programme in T2D patients.

Preliminary results from this study indicate some differences in stature and corpulence categories. The majority of men were overweight while most women were rather obese. Depending on the stages of life, there is a considerable variation in adiposity and metabolism between the sexes. Several clinical studies confirm gender differences in fat storage, fat cell modelling / reshaping, and lipolytic responses. ${ }^{21}$ According to the age of our patients (49.83 \pm 12.24 years for women), it is probable that most of female patients are post-menopausal. The higher level of oestrogen protect postmenopausal women comparing to men and premenopausal women. However, higher androgen levels can be detrimental for females and beneficial for men. ${ }^{22}$

In the present study, women with type 2 diabetes had higher fasting blood glucose, HbA1c, total cholesterol, HDL and LDL levels than their male counterparts. In contrast, men had higher triglyceride levels, kidney function parameters (urea and creatinine) and all lipid ratios (TC/HDL, LDL/HDL and TG/HDL). This non-significant inconsistency was different to the findings of Aderibigbe et al.; women with type 2 diabetes have generally poorer kidney function and a more disturbed lipid profile than their male counterparts. ${ }^{23}$ It should be noted that our findings indicate a non-significant $(p>0.05)$ effect of gender on major biochemical parameters. Poehlman et al. ${ }^{24}$ suggested the same conclusions.

The LDL/HDL ratio and the TC/HDL ratio (atherogenic or Castelli index) are important vascular risk indicators with greater predictive value comparing to the traditional isolated lipid parameters used independently. ${ }^{25}$ In the Helsinki Heart Study, it was demonstrated that the LDL/HDL ratio is a better cardiovascular risk indices in the presence of hypertriglyceridemia. ${ }^{26}$

In the present study, the level of LDL/HDL is $<2.5$ for both genders, while the target level for men and women are 3.0 and 2.5 , respectively in primary intervention. ${ }^{23}$ The TC/HDL ratio value was below the target level of 4.5 and 4.0 for men and women. However, the TG/HDL ratio or the atherogenic plasma index (AIP) is employed as an additional index when evaluating cardiovascular risk factors. The values of the TG/HDL ratio of our population are lower than the target value of 2.5 in both sexes. ${ }^{27,} 28$ All our lipid ratios values suggested a low cardiovascular risk in our T2D patients.

Results of the current study revealed a positive impact of the physical activity program during 28 weeks on the studied variables, for both men and women. All the biochemical, clinical and anthropometric parameters linked to cardiometabolic risk were improved with the brisk walking (30 min/day, 3 times a week), and those from month to month until the seventh month (end of the study).
Results similar to those obtained here were reported in several studies. It has been firmly established that physical activity or ${ }^{29}$ participation in structured exercise intervention programs ${ }^{19,} 30$ improves glycaemic control. Higher significant decreases in fasting, post-effort and postprandial glycaemia were observed in men and women T2D patients. However, according to several authors, the absence of a significant drop in HbA1c blood levels in brisk walkers does not mean that this type of low-impact endurance physical activity has no therapeutic value. ${ }^{19,}$, 30-33

In our patients, body composition (weight and BMI), traditional lipid profile and metabolic risk indices (lipid ratios) were improved. Our results are similar to the conclusions of Aggarwala et al. who reported that exercise improves diabetic status and reduces metabolic risk factors, insulin sensitivity and lipid profile. ${ }^{34}$ They recommended an aerobic exercise program of at least $150 \mathrm{~min} /$ week of aerobic physical activity spread over at least 3 days/week with no more than 2 consecutive days without exercise.

The outcomes of numerous Meta-analyses about the effect of aerobic physical activity on lipid and lipoprotein levels in participants with T2D are discordant. For example, Yoo \& Lee found a positive effect of regular exercise on TC, HDL, and LDL in T2D patients, ${ }^{35}$ Kelley \& Kelley found that aerobic exercise reduced LDL rates alone, ${ }^{36}$ while Thomas et al. reported statistically significant reductions in TG but not TC, HDL or LDL. ${ }^{37}$

On the other hand, the effect of brisk walking on the improvement of aerobic capacity was not conclusive since we recorded a non-significant fluctuation in $\mathrm{VO}_{2}$ max and basic heart rate during the seven months of the study in the two genders. In fact, according to bibliographic data, aerobic exercise has a positive impact on increasing oxygen absorption and the oxygen use in skeletal muscle, increasing skeletal muscle oxidative capacity, and, improving glycaemic control through a good insulin sensitivity. ${ }^{38}$

Lipid ratios (TC/HDL, LDL/HDL and TG/HDL) at the start (1 1 st month) and the completion ( $7^{\text {th }}$ month) of the study were compared between males and females according to diabetic control profile estimated through HbA1c levels (HbA1c $\leq 7 \%$ vs. HbA1c> 7\%). Currently, HbA1c levels are established as the gold standard for glycaemic control in diabetic patients. A level of $\mathrm{HbA} 1 \mathrm{c} \leq 7 \%$ is generally linked to a reduced risk of cardiovascular complications. ${ }^{39}$ In our study, the diabetic patients (both males and females) with $\mathrm{HbA1c}$ value $>7 \%$ do not exhibited a significant difference of lipid ratios (TC/HDL, LDL/HDL and TG/HDL) when comparing to T2D patients with $\mathrm{HbA1} \leq 7 \%$. However, we noted a non-significant decrease in lipid ratios values when comparing the $1^{\text {st }}$ to the $7^{\text {th }}$ month results in both men and women. These conclusions are consistent with some previous studies. $40-42$ The hyperlipidaemia observed in our female patients can be attributed to the effects of sex hormones on body fat distribution, which leads to differences in changed lipoproteins. 42,43

For better interpretation of the impact of brisk walking program (in the two genders) on $\mathrm{VO}_{2}$ max, BMI and Heart Rate according to the HbA1c level, we compared these parameters between the $1^{\text {st }}$ and the $7^{\text {th }}$ month. No significant differences were observed between the "beginning" and the "end" of the study regarding the $\mathrm{VO}_{2}$ max, BMI and Heart Rate whether between the two genders or between the two levels of HbA1c. Our results are different from several studies conclusions showing that maximal oxygen consumption $\left(\mathrm{VO}_{2} \mathrm{max}\right)$ and $\mathrm{BMI}$ improve favourably after an aerobic physical activity program. ${ }^{44-46}$ 
Our study is not without limitations. The small sample size and the absence of control group (subjects without diabetes or sedentary) do not allow to make a clear comparison between groups. Another limitation of the study is that we do not have information about the nutritional status of the participants while this parameter is essential in the management of the diabetic disease and its associated complications. However, despite these limitations we believe that our conclusions would remain unchanged in the general population since we considered each patient as his own control through the comparison of the studied parameters during a long period ( 7 months $=28$ weeks). The nutritional evaluation could not be constant during the entire study period and the evaluation methods will therefore be very heavy and difficult to realize.

\section{CONCLUSION}

Physical activity remains a key element in the prevention and management of type 2 diabetes and its complications. However, many people with this disease do not become or do not remain regularly active due to the multiplicity, the intensity or even the cost of collective sports sessions. The findings of the current study indicate that adherence to physical activities of moderate intensity such as brisk walking can substantially be a good alternative. The practice of three brisk walking sessions per week at a rate of 30 minutes each has beneficial impact on anthropometric parameters, biochemical parameters, and physical performance and on the reduction of cardiovascular risk in patients with type 2 diabetes of both genders.

\section{ACKNOWLEDGEMENTS}

Our gratitude is addressed to all the patients who participated in the study for their patience and punctuality.

\section{Conflict of Interest: No}

\section{REFERENCES}

1. American Diabetes Association, "Classification and diagnosis of diabetes" Diabetes Care, 2016; 39(suppl 1):S13-S22. doi: $10.2337 /$ dc16-S005.

2. Ogurtsova K, da Rocha Fernandes JD, Huang Y, Linnenkamp U, Guariguata L, Cho NH, Cavan D, Shaw JE, Makaroff LE, "IDF Diabetes Atlas: Global estimates for the prevalence of diabetes for 2015 and 2040" Diabetes Res Clin Pract, 2017; 128:40-50. doi: 10.1016/j.diabres.2017.03.024.

3. Petrie JR, Guzik TJ, Touyz RM, "Diabetes, Hypertension, and Cardiovascular Disease: Clinical Insights and Vascular Mechanisms" Can J Cardiol, 2018; 34(5):575-584. doi:10.1016/j.cjca.2017.12.005.

4. Garber AJ, Abrahamson MJ, Barzilay JI, Blonde L, Bloomgarden ZT, Bush MA, Dagogo-Jack S, DeFronzo RA, Einhorn D, Fonseca VA, Garber JR, Garvey WT, Grunberger G, Handelsman Y, Hirsch IB, Jellinger PS, McGill JB, Mechanick JI, Rosenblit PD, Umpierrez GE, "Consensus statement by the american association of clinical endocrinologists and american college of endocrinology on the comprehensive type 2 diabetes management algorithm - 2018 executive summary" Endocr Pract, 2018; 24(1):91-120. doi: 10.4158/CS-2017-0153.

5. American Diabetes Association, "Cardiovascular disease and risk management: standards of medical care in diabetes-2018" Diabetes Care, 2018; 41(suppl 1):S86-S104. doi: 10.2337/dc18-S009.

6. Sherr D, Lipman RD, "The diabetes educator and the diabetes self-management education engagement" Diabetes Educ, 2015; 41(5):616-624.

7. US Department of Health and Human Services, Centers for Disease Control and Prevention. Nutrition, Physical Activity and Obesity Data, Trends, and Maps Website. 2015. Available at: https://www.cdc.gov/nccdphp/dnpao/data-trendsmaps/index.html.

8. Buresh R, Berg K, "Exercise for the management of type 2 diabetes mellitus: factors to consider with current guidelines" J Sports Med Phys Fitness, 2018; 58(4):510-524. doi:10.23736/S0022-4707.17.06969-9.

9. Colberg SR, Sigal RJ, Fernhall B, Regensteiner JG, Blissmer BJ, Rubin RR, Chasan-Taber L, Albright AL, Braun B; American College of Sports Medicine; American Diabetes Association, "Exercise and type 2 diabetes: the American College of Sports Medicine and the American Diabetes Association: joint position statement" Diabetes Care, 2010; 33(12):e147-e167. doi: $10.2337 / \mathrm{dc} 10-9990$.

10. Gusso S, Hofman P, Lalande S, Cutfield W, Robinson E, Baldi JC, "Impaired stroke volume and aerobic capacity in female adolescents with type 1 and type 2 diabetes mellitus" Diabetologia, 2008; 51(7):1317-1320. doi: 10.1007/s00125008-1012-1.

11. Weinstock RS, Brooks G, Palmas W, Morin PC, Teresi JA, Eimicke JP, Silver S, Izquierdo R, Goland R, Shea S, "Lessened decline in physical activity and impairment of older adults with diabetes with telemedicine and pedometer use: results from the IDEATel study" Age Ageing, 2011; 40(1):98-105. doi: 10.1093/ageing/afq147.

12. Thomas N, Alder E, Leese GP, "Barriers to physical activity in patients with diabetes" Postgrad Med J, 2004; 80(943):287291. doi:10.1136/pgmj.2003.010553.

13. Qiu S, Cai X, Schumann U, Velders M, Sun Z, Steinacker JM, "Impact of walking on glycemic control and other cardiovascular risk factors in type 2 diabetes: a metaanalysis" PLoS One, 2014; 9(10):e109767. doi: 10.1371/journal.pone.0109767.

14. Trivel D ; Leger L ; Calmels P, "Estimation de l'aptitude physique par questionnaire Fitness assessment by questionnaire" Science Sports, 2006; 21(3):121-130. https://doi.org/10.1016/j.scispo.2005.12.004.

15. BoussanaA, Hue 0 , Hayot $M$, Matécki $S$, Ramonatxo M, Le Gallais D, "Capacité de diffusion pulmonaire avant un triathlon et 24 heures après la compétitionPulmonary diffusing capacity before and $24 \mathrm{~h}$ after a triathlon" Science \& Sports, 2000; 15(5):245-247.

16. Berger $M$, "Die Muskelarbeit in der Therapie des Diabetes mellitus [Muscular work in the therapy of diabetes mellitus]" Fortschr Med, 1976; 94(28):1553-1557.

17. Conn VS, Hafdahl AR, Mehr DR, LeMaster JW, Brown SA, Nielsen PJ, "Metabolic effects of interventions to increase exercise in adults with type 2 diabetes" Diabetologia, 2007; 50(5):913-921. doi: 10.1007/s00125-007-0625-0.

18. Burns N, Finucane FM, Hatunic M, Gilman M, Murphy M, Gasparro D, Mari A, Gastaldelli A, Nolan JJ, "Early-onset type 2 diabetes in obese white subjects is characterised by a marked defect in beta cell insulin secretion, severe insulin resistance and a lack of response to aerobic exercise training" Diabetologia, 2007; 50(7):1500-1508. doi: 10.1007/s00125007-0655-7.

19. Praet SF, van Rooij ES, Wijtvliet A, Boonman-de Winter LJ, Enneking T, Kuipers H, Stehouwer CD, van Loon LJ, "Brisk walking compared with an individualised medical fitness programme for patients with type 2 diabetes: a randomised controlled trial" Diabetologia, 2008; 51(5):736-746. doi: 10.1007/s00125-008-0950-y.

20. Hamasaki H, "Daily physical activity and type 2 diabetes: A review" World J Diabetes, 2016; 7(12):243-251. doi:10.4239/wjd.v7.i12.243

21. Cancello R, Tordjman J, Poitou C, Guilhem G, Bouillot JL, Hugol D, Coussieu C, Basdevant A, Bar Hen A, Bedossa P, GuerreMillo M, Clément $\mathrm{K}$, "Increased infiltration of macrophages in omental adipose tissue is associated with marked hepatic lesions in morbid human obesity" Diabetes, 2006; 55(6):15541561. doi: 10.2337/db06-0133.

22. Chang E, Varghese M, Singer K, "Gender and Sex Differences in Adipose Tissue" Curr Diab Rep, 2018; 18(9):69. doi:10.1007/s11892-018-1031-3.

23. Aderibigbe MA, Obafemi TO, Olaleye MT, Akinmoladun AC, "Effects of gender, age and treatment duration on lipid profile and renal function indices in diabetic patients attending a teaching hospital in South-Western Nigeria" Afr Health Sci, 2018; 18(4):900-908. doi:10.4314/ahs.v18i4.8.

24. Poehlman ET, Toth MJ, Ades PA, Rosen CJ, "Menopauseassociated changes in plasma lipids, insulin-like growth factor I and blood pressure: a longitudinal study" Eur J Clin Invest, 1997; 27:322-326. 
25. Millán J, Pintó X, Muñoz A, Zúñiga M, Rubiés-Prat J, Pallardo LF, Masana L, Mangas A, Hernández-Mijares A, GonzálezSantos P, Ascaso JF, Pedro-Botet J, "Lipoprotein ratios: Physiological significance and clinical usefulness in cardiovascular prevention" Vasc Health Risk Manag, 2009; 5:757-765.

26. Manninen V, Tenkanen L, Koskinen P, Huttunen JK, Mänttäri $\mathrm{M}$, Heinonen OP, Frick MH, "Joint effects of serum triglyceride LDL-C cholesterol and HDL-C cholesterol concentrations on coronary heart disease risk in the Helsinki Heart Study. Implications for treatment" Circulation, 1992; 85(1):37-45.

27. Dobiasova M, Frohlich J, "The plasma parameter log (TG/HDLC-C) as an atherogenic index: correlation with lipoprotein particle size and esterification rate in apo B- lipoproteindepleted plasma. (FERHDL-C)" Clinical Biochemistry, 2001; 34(7):583-588.

28. Tan MH, Johns D, Glazer NB, "Pioglitazone reduces atherogenic index of plasma in patients with type 2 diabetes" Clinical Chemistry, 2004; 50(7):1184-1188.

29. Kirk A, Mutrie N, MacIntyre P, Fisher M, "Effects of a 12-month physical activity counselling intervention on glycaemic control and on the status of cardiovascular risk factors in people with Type 2 diabetes" Diabetologia, 2004; 47(5):821-832. doi: 10.1007/s00125-004-1396-5.

30. Loimaala A, Huikuri HV, Koobi T, Rinne M, Nenonen A, Vuori I "Exercise training improves baroreflex sensitivity in type 2 diabetes". Diabetes, 2003; 52:1837-1842.

31. Snowling NJ, Hopkins WG, "Effects of different modes of exercise training on glucose control and risk factors for complications in type 2 diabetic patients: a meta-analysis" Diabetes Care, 2006; 29:2518-2527.

32. Sigal RJ, Kenny GP, Wasserman DH, Castaneda-Sceppa C, White RD, "Physical activity/exercise and type 2 diabetes: a consensus statement from the American Diabetes Association" Diabetes Care, 2006; 29:1433-1438.

33. Pedersen BK, Saltin B, "Evidence for prescribing exercise as therapy in chronic disease" Scand J Med Sci Sports, 2006; 16(Suppl 1):3-63.

34. Aggarwala J, Sharma S, Saroochi, Jain A, Sarkar A, "Effects of aerobic exercise on blood glucose levels and lipid profile in Diabetes Mellitus type 2 subjects" Al Ameen J Med Sci, 2016; 9(1):65-69.

35. Yoo JS, Lee SJ, A meta-analysis of the effects of exercise programs on glucose and lipid metabolism and cardiac function in patients with type II diabetes mellitus [in Korean]" Taehan Kanho Hakhoe Chi, 2005; 35:546-554.

36. Kelley GA, Kelley KS, "Effects of aerobic exercise on lipids and lipoproteins in adults with type 2 diabetes: a meta-analysis of randomized-controlled trials" Public Health, 2007; 121(9):643-655. doi:10.1016/j.puhe.2007.02.014

37. Thomas D, Elliott EJ, Naughton GA, "Exercise for type 2 diabetes mellitus" Cochrane Database of Systematic Reviews,
2006; (3), Art. No.: CD002968. doi: 10.1002/14651858.CD002968.pub2.

38. Nojima H, Yoneda M, Watanabe H, Yamane $K$, Kitahara Y, Sekikawa K, Yamamoto H, Yokoyama A, Hattori N, Kohno N; Hiroshima University Health Promotion Study group, "Association between aerobic capacity and the improvement in glycemic control after the exercise training in type 2 diabetes" Diabetol Metab Syndr, 2017; 9:63. doi: 10.1186/s13098-017-0262-9.

39. Rohlfing CL, Wiedmeyer HM, Little RR, England JD, Tennill A, Goldstein DE, "Defining the relationship between plasma glucose and HbA1c: analysis of glucose profiles and HbA1c in the Diabetes Control and Complications trial" Diabetes Care, 2002; 25:275-278.

40. VinodMahato R, Gyawali P, Raut PP, Regmi P, Singh KP, Raj Pandeya D, Gyawali P, "Association between glycaemic control and serum lipid profile in type 2 diabetic patients: glycated haemoglobin as a dual biomarker" Biomed Res, 2011; 22: 375380.

41. Dixit AK, Dey R, Suresh A, Chaudhuri S, Panda AK, Mitra A, Hazra J, "The prevalence of dyslipidemia in patients with diabetes mellitus of ayurveda Hospital" J Diabetes Metab Disord, 2014;13:58. doi: 10.1186/2251-6581-13-58.

42. Hussain A, Ali I, Ijaz M, Rahim A, "Correlation between hemoglobin A1c and serum lipid profile in Afghani patients with type 2 diabetes: hemoglobin A1c prognosticates dyslipidemia" Ther Adv Endocrinol Metab, 2017; 8(4):51-57. doi: $10.1177 / 2042018817692296$.

43. Sibley SD, Thomas W, de Boer I, Brunzell JD, Steffes MW, "Gender and elevated albumin excretion in the Diabetes Control and Complications Trial/Epidemiology of Diabetes Interventions and Complications (DCCT/EDIC) cohort: role of central obesity" Am J Kidney Dis, 2006; 47(2):223-32. doi: 10.1053/j.ajkd.2005.10.021.

44. Balducci S, Zanuso S, Nicolucci A, De Feo P, Cavallo S, Cardelli P, Fallucca S, Alessi E, Fallucca F, Pugliese G; Italian Diabetes Exercise Study (IDES) Investigators, "Effect of an intensive exercise intervention strategy on modifiable cardiovascular risk factors in subjects with type 2 diabetes mellitus: a randomized controlled trial: the Italian diabetes and exercise study (IDES)" Arch Intern Med, 2010; 170:1794-1803.

45. Sigal RJ, Kenny GP, Boule NG, Wells GA, Prud'homme D, Fortier M, Reid RD, Tulloch H, Coyle D, Phillips P, Jennings A, Jaffey J, "Effects of aerobic training, resistance training, or both on glycemic control in type 2 diabetes: a randomized trial" Ann Intern Med, 2007; 147:357-369.

46. Wang T, Liu Y, Zhong R, Xu D, Wang H, Fu BSC, "Benefit effects of aerobic exercise and resistance training on the management of type 2 diabetes" Int J Clin Exp Med, 2018; 11(10):1043310445 . 